\title{
Radio Wave Characterization in Wireless Sensor Network-Based Intelligent Transportation Systems for Traffic Light Control Systems
}

\section{Carlos Martínez $^{1 *}$, Leyre Azpilicueta ${ }^{1,3}$, Peio López-Iturri ${ }^{1}$, Erik Aguirre ${ }^{1}$, José Javier Astráin ${ }^{2,3}$, Jesús Villadangos ${ }^{2,3}$ and Francisco Falcone ${ }^{1,3}$}

1 Electrical and Electronic Engineering Department, Universidad Pública de Navarra, Pamplona 31006, Spain; E-Mails: martinez.61368@e.unavarra.es (C.M); leyre.azpilicueta@unavarra.es (L.A.); peio.lopez@unavarra.es (P.L.); aguirrerik@gmail.com (E.A.); francisco.falcone@unavarra.es (F.F.)

2 Mathematical Engineering and Computer Science Department, Universidad Pública de Navarra, Pamplona 31006, Spain; E-Mails: josej.astrain@unavarra.es (J.J.A.); jesusv@unavarra.es (J.V.)

3 Institute for Smart Cities, Universidad Pública de Navarra, Pamplona 31006, Spain

* Author to whom correspondence should be addressed; E-Mail: leyre.azpilicueta@unavarra.es (L.A.); Tel.: +34-948-169-095; Fax: +34-948-169-720.

Published: 9 November 2015

\begin{abstract}
With the growing demand of Intelligent Transportation Systems (ITS) for safer and more efficient transportation, the research and development of such vehicular communication systems have increased considerably in the last years. In this sense, wireless systems have been increasingly adopted in order to enable ITS communication. In this context, adequate wireless channel characterization is compulsory on order to guarantee quality of service requirements. In this work, the radio wave characterization for ISM $2.4 \mathrm{GHz}$ Wireless Sensor Networks (WSN) for a traffic light control system has been assessed. By means of an in-house developed 3D Ray Launching algorithm, the impact of topology as well as morphology of the environment has been analyzed, emulating a realistic Traffic Light system in an urban placement. The complexity of the scenario, which is an intersection city area with traffic lights, vehicles, people, buildings, vegetation and urban environment, makes necessary the channel characterization with accurate models before the deployment of wireless networks. A measurement campaign has been conducted emulating the traffic light control system. Results show that the use of deterministic tools can aid in providing optimal network deployment to fulfill service requirements of the required WSN to enable interactive Traffic Light system.
\end{abstract}


Keywords: traffic lights; propagation; wireless sensor networks; ray launching.

\section{Introduction}

In the past two decades, the number of vehicles circulating in cities has increased considerably. This, together with the tendency of citizens to clustering has caused congestions of roads and junctions, especially in cities with large populations. The existing infrastructures have become inefficient to optimize traffic. The traditional traffic control system based on traffic lights depending on the preset date and time patterns is not efficient in reducing waiting times and energy consumption. In fact, simulations show that adaptive traffic based on information from Wireless Sensor Networks (WSNs) can be reduced by $65 \%$ in normal traffic conditions regarding traffic management by preset patterns [1]. Operation of Wireless Systems in general requires to achieve signal levels at all nodes above minimum receiver thresholds, which mainly depends on propagation losses and hence, adequate loss estimation, provided by radio-planning techniques. There are several techniques which can be employed, from semiempirical approaches, with low computational cost but limited accuracy up to full wave simulation results, which provide high resolution at a very high computational cost. As a midpoint, deterministic methods, provided by Ray Launching and Ray tracing techniques, based on geometric optics, provide an adequate trade-off between accuracy and computational complexity [2-3].

In this work, wireless channel performance as a function of propagation losses has been analyzed applied to an urban area for an adaptive traffic light control system, with the aim to optimize inter-nodal distance and location.

\section{Ray Launching Technique}

In order to perform radio propagation analysis of the traffic light system, a 3D Ray Launching algorithm implemented in-house has been employed [4-5]. The 3D RL code is based on a geometric optics approximation, combined with Uniform Theory of Diffraction, in which rays are launched from a given radiation sources, with a number of rays launched as a function of angular resolution in both theta and phi angular planes, providing effective solid angle operation. Interaction with the surrounding elements of the environment is taken into account by considering frequency dispersive material parameters, as well as refraction, reflection and diffraction phenomena, as a function of system operation frequency. Additional parameters such as required antenna radiation patterns, cuboid resolution and number of reflections up to ray extinction are also considered. Figure 1 shows the real and schematic view of the considered scenario. The scenario consists of an outdoor environment of an urban city area, with trees of different heights, grass, junctions of roads, cars, people and different type of buildings. This environment can be considered as really complex, in terms of radio wave propagation, because all the elements with different material properties within it. The dimensions of the scenario are $(150 \mathrm{~m}, 130 \mathrm{~m}$, $30 \mathrm{~m})$. 


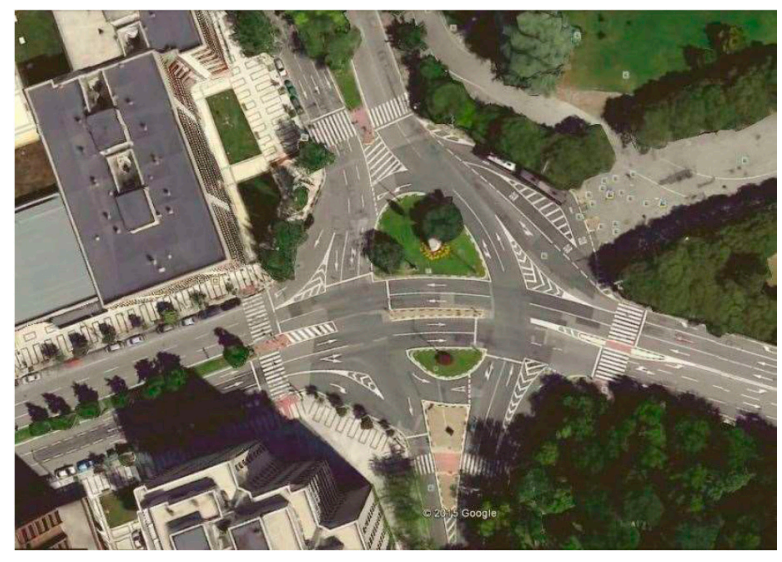

(a)

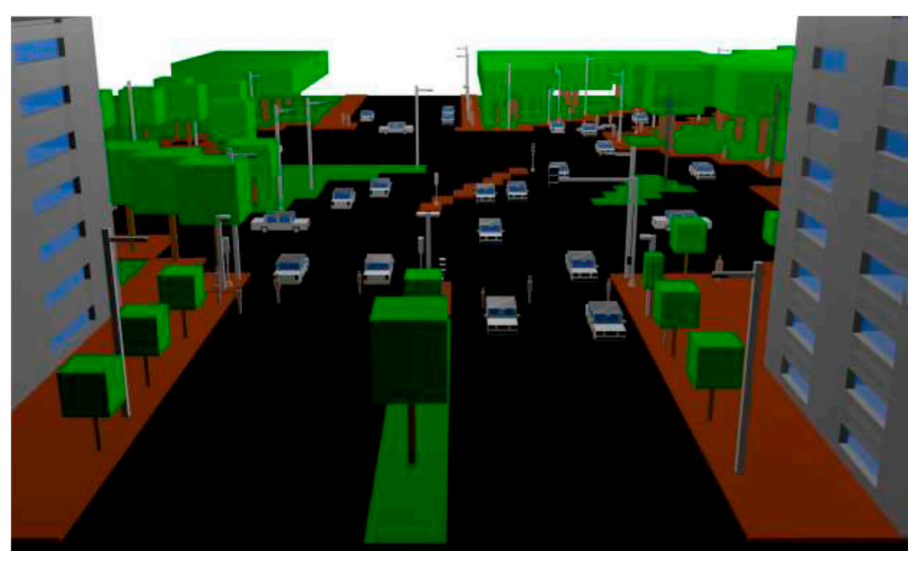

(b)

Figure 1. Implemented scenario to analyze wireless channel behavior for the Traffic Light system (a) Real scenario (b) Schematic view of the scenario.

For the simulations, 16 antennas have been placed in the considered scenario, one for each traffic light. To reduce interferences with people and vehicles, each antenna has been placed $10 \mathrm{~cm}$ above each traffic light. The considered transceivers are ZigBee motes with a dipole antenna, transmitting $18 \mathrm{dBm}$ at $2.41 \mathrm{GHz}$. Simulation parameters are shown in Table 1.

Table 1. Simulation Parameters for the Traffic Light Scenario.

\begin{tabular}{lll}
\hline FREQUENCY OF OPERATION & & $2.41 \mathrm{GHz}$ \\
VERTICAL PLANE ANGLE RESOLUTION & $\Delta \theta$ & $2^{\circ}$ \\
\hline HORIZONTAL PLANE ANGLE RESOLUTION & $\Delta \varphi$ & $2^{\circ}$ \\
MAXIMUM \# REFLECTIONS & & 6 \\
TRANSCEIVER POWER & & $18 \mathrm{DBM}$ \\
CUBOIDS RESOLUTION & & $2 \mathrm{M}$ \\
\hline
\end{tabular}

\section{Results and Discussion}

The results depicted in Figure 2 correspond to the received power distribution within the Traffic Light scenario, for different height cut planes. Noticeable differences can be observed provided that different object distributions are present as the location of the potential transceivers change (i.e., topological and morphological changes in the distribution of elements such as urban furnishings, street lights, buildings, vegetation, etc.).
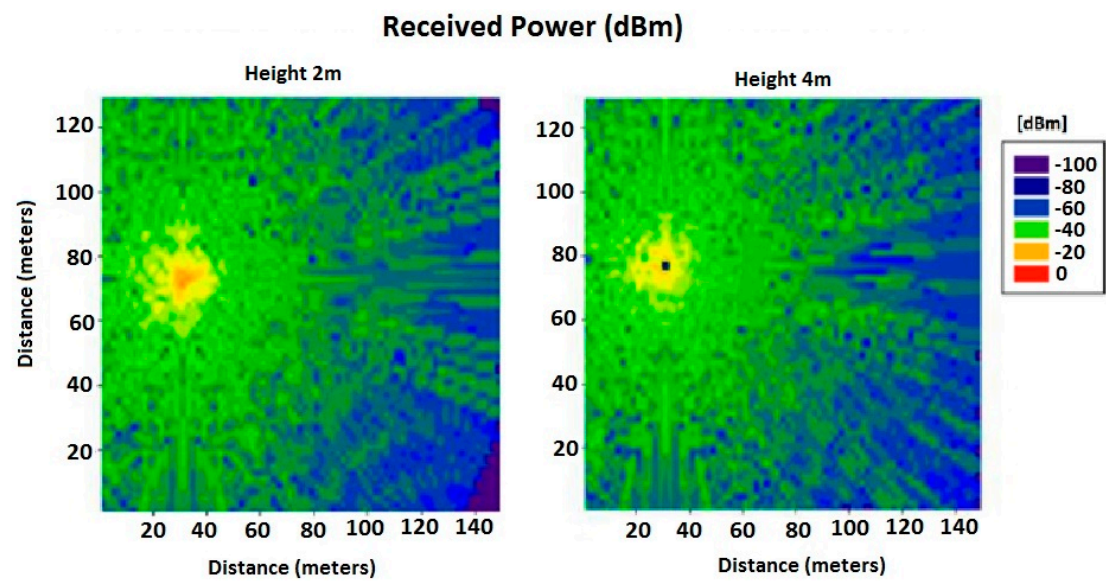
Figure 2. Received power level estimation values $(\mathrm{dBm})$ on the considered at the Traffic Light scenario for different observation heights.
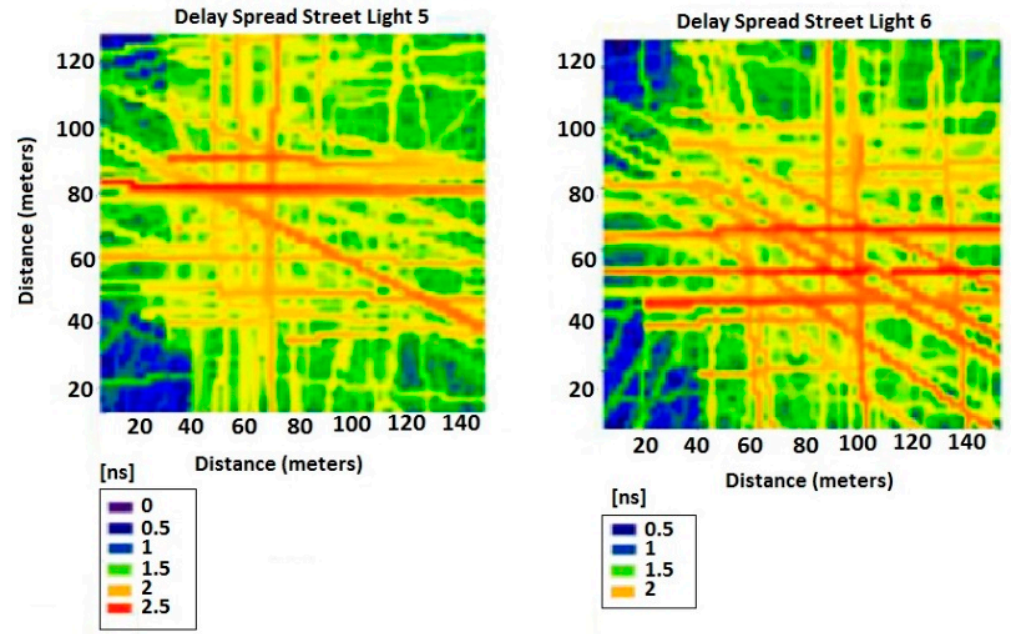

Figure 3. Estimation of delay spread (ns) on the considered scenario for different positions of the transmitter antenna in different street lights.

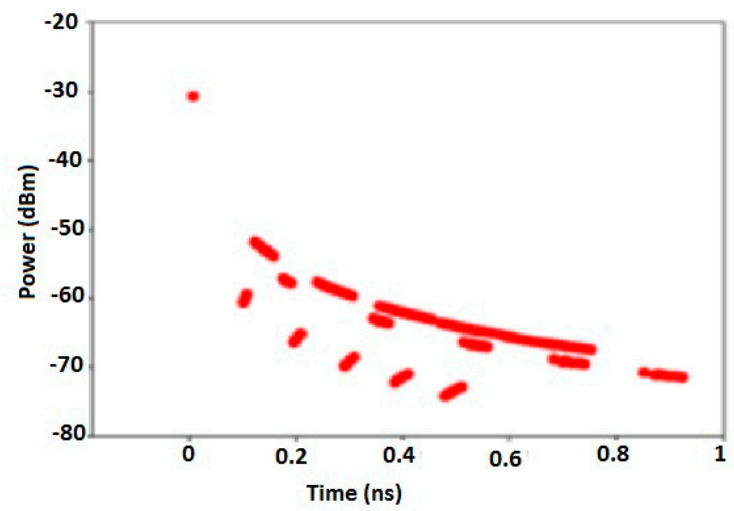

Figure 4. Estimation of Power Delay Profile, for a cuboid at a central location of the simulation scenario.

One of the main characteristics of a complex urban environment is the predominant role of multipath propagation, which arises from the multiple reflections of propagating waves with all the elements which are present within the scenario. In order to illustrate this fact, Power Delay Profile estimations, which consider the time domain distribution of direct transmitted components as well as echoes derived from such reflections, are shown in Figure 3 and Figure 4. As it can be seen, the distribution provides multiple echoes within the cuboid under analysis, in direct relation with the complex nature of the environment in terms of radioelectric propagation.

\section{Application}

This paper focuses on Smart Cities, and taking into account that about 15\% (about 1 billion people) of the world's population has any kind of disability, we have developed an application devoted to minimize the impedance between these citizens and the infrastructures and services of the cities. 


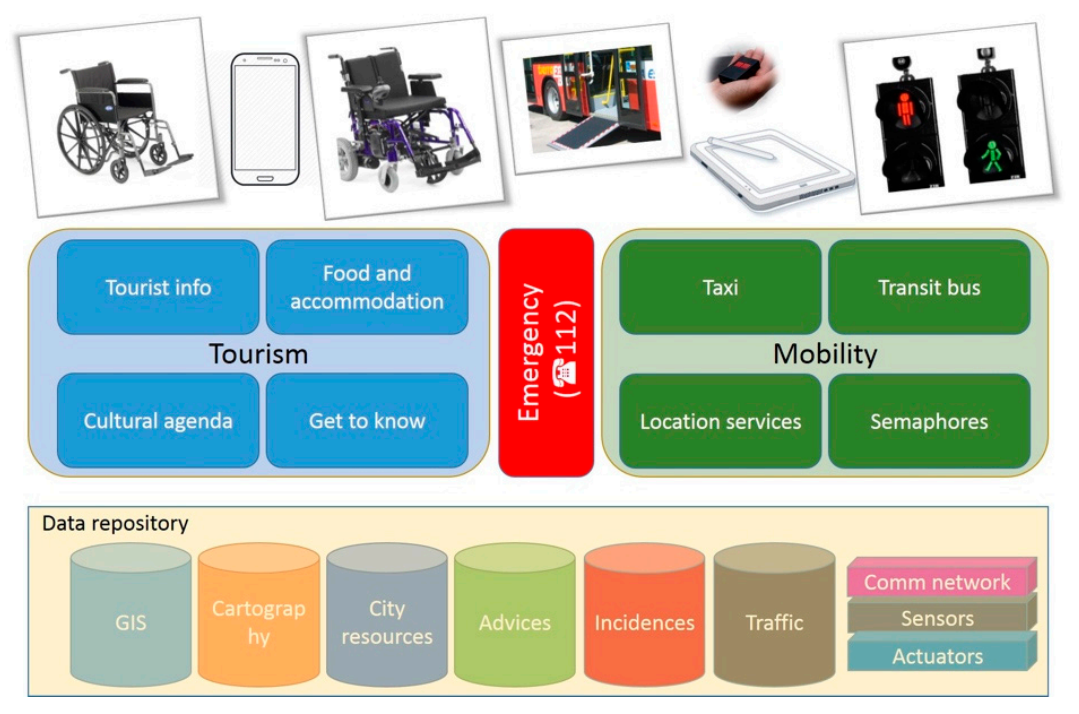

Figure 5. System architecture: devices (top), services (middle) and repositories/infrastructure (bottom).

First, we have identified different user profiles according to the type and degree of disability the citizens have. This will allow providing the best possible range of solutions to each of the issues that the citizen can found in her/his daily lives. Thus, if a blind user approaches a crosswalk, it would be interesting, as far as possible, that infrastructure was aware of the arrival of the citizen activating the acoustic signals of the traffic light. In the same way, if a citizen traveling in a wheelchair approaches a bus stop in order to take a specific urban bus, the infrastructure may notified the bus so that it deploys its hydraulic ramp at its arrival and advises the rest of users in order to facilitate to the citizen the access to the vehicle. The system architecture adopted is a service-based architecture where each user can subscribe different services according to her/his disability and preferences. Some of the services offered follow a push model (announces concerning emergences, work/repair tasks, weather conditions...) while others follow a pull model (taxi request, a green stoplight request...). The services are available through the personal smartphones of users and are integrated on special devices as intelligent wheelchairs. Figure 5 describes the architecture of the system.

Since wireless communication is the key issue of this architecture, an RF characterization of the scenario is essential in order to ensure the success of the project. The location services used to provide services are based on wireless technologies and require a wide study.

\section{Conclusions}

In this work, surrounding environment impact in the operation of wireless channels in an urban area for intelligent traffic light control systems is analyzed, which exhibit intrinsic complexity, due to the large amount of elements within the scenario. With the aid of deterministic 3D Ray Launching simulation tools, precise characterization of wireless channel behavior can be obtained, which leads to optimal transceiver location, in order to minimize energy consumption, minimize interference and maximize overall quality of service in complex large scale wireless sensor networks, applicable in the case of Traffil Light systems in urban environments. 


\section{Author Contributions}

Carlos Martínez, Leyre Azpilicueta, Erik Aguirre, Peio López-Iturri and Francisco Falcone conducted the characterization of wireless propagation mechanisms in the Traffic Light scenario. José Javier Astráin and Jesús Villadangos participated in the development of the WSN and the application layer for such scenario.

\section{Conflicts of Interest}

The authors declare no conflict of interest.

\section{References}

1. Abb-Fatah, A. Y., Yusuff, R. M., Aziz, F. A. and Zulkifli, N. Simulation of time based versus sensor based traffic light system. IEEE $3^{\text {rd }}$ International Conference on the Communication Software and Networks (ICCSN), 2011.

2. Tubaishat, M., Yi, S. and Hongchi, S. Adaptive Traffic Light Control with Wireless Sensor Networks. $4^{\text {th }}$ IEEE Consumer Communications and Networking Conference, 2007.

3. Fuqiang, Z., Bo, Y. and Yitao, C. Traffic light control for a single intersection based on wireless sensor network. $9^{\text {th }}$ International Conference on Electronic Measurement and Instrumentation.

4. Azpilicueta, L., Falcone, F., Astráin, J. J., Villadangos, J., García Zuazola, I. J., Landaluce, H. Angulo, I., Perallos, A. Measurement and Modeling of a UHF-RFID System in a Metallic Closed Vehicle. Microwave and Optical Technology Letters, 2012, vol. 54, nº 9, pp. 2126-2130.

5. Elejoste P., Angulo, I. Perallos, A., Chertudi, A., García Zuazola, I. J., Moreno, A., Azpilicueta, L., Astráin, J. J., Falcone, F. An Easy to Deploy Street Light Control System Based on Wireless Communication and LED Technology. Sensors, 2013, vol. 13, pp. 6492-6523.

(C) 2015 by the authors; licensee MDPI, Basel, Switzerland. This article is an open access article distributed under the terms and conditions of the Creative Commons Attribution license (http://creativecommons.org/licenses/by/4.0/). 